\title{
Damage Function and Its Application
}

\author{
Andrey Tarantsev ${ }^{1}$, Igor Babikov ${ }^{2}$, Dan Savelyev ${ }^{2 *}$, Mikhail Vasiliev², and Denis \\ Raspopov $^{3}$ \\ ${ }^{1}$ Academy of the State Fire Service of Emercom of Russia, 129366, Boris Galushkin st, 4, Moscow, \\ Russia \\ ${ }^{2}$ Peter the Great St. Petersburg Polytechnic University, 195521 Polytechnicheskaya, 29, Saint- \\ Petersburg, Russia \\ ${ }^{3}$ FSBEE HE «Saint-Petersburg University of State Fire Service of EMERCOM of Russia», 196105, \\ Moskovsky prospect, 149, Saint-Petersburg, Russia
}

\begin{abstract}
The types of damage functions for objects in the event of various emergency situations - fires, accidents, emergencies, etc. - are considered. The methods of constructing damage functions using expert methods and taking into account the actions of emergency services to eliminate emergency situations and their consequences are presented. The possibility of comparing the effectiveness of various methods of emergency response using the damage function is shown. An example of assessing damage reduction while increasing the efficiency of fire extinguishing by automatic installations is provided.
\end{abstract}

\section{Introduction}

In the event of emergency situations (fires, accidents, emergencies, etc.) at industrial [1] and other facilities, they are usually damaged. At the same time, the amount of damage depends both on the object itself and on the type of emergency situation and measures to minimize it - on the effectiveness of fire extinguishing, emergency rescue operations (ERO) [2], etc. When developing planning documents [3-5] and risk assessment [6, 7] is of interest to determine the dynamics of possible damage, for which it was proposed $[8,9]$ to use a special curve $U(t)$, called the «damage function».

\section{Materials and methods}

The initial damage function $U(t)$ is a curve that monotonically increases from $U=0$ (at time $t=0$ of the onset of an emergency situation, there is still no damage) to $U=1$, when the damage reaches its greatest value (Fig. 1).

\footnotetext{
* Corresponding author: savelev.dan@yandex.ru
} 


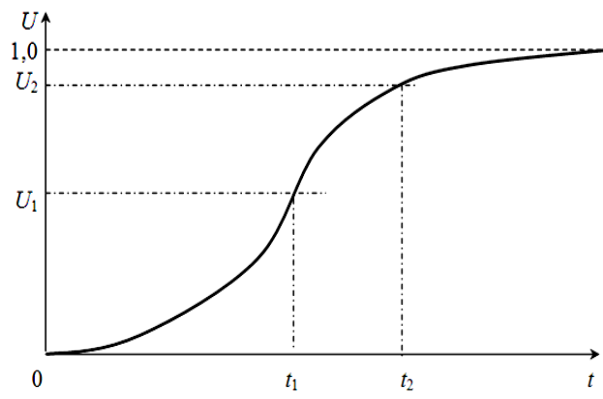

a)

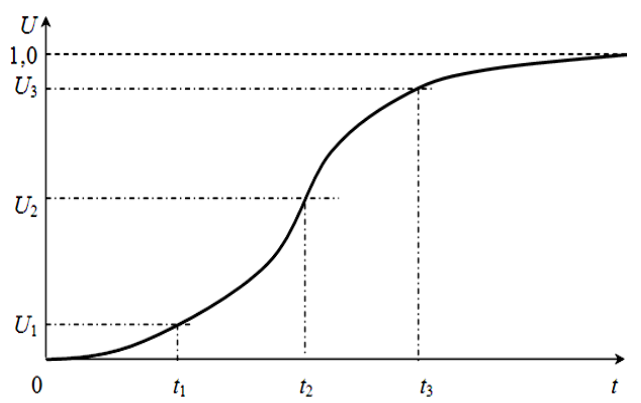

b)

Fig. 1. Initial damage function: a) when represented by 2 points, $b$ ) when represented by 3 points

Mathematical expressions for the function $U(t)$ that meet the requirements for it can have different forms (for example, $[8,9]$ ):

$$
\begin{aligned}
& U(t)=\left(1-e^{-k t}\right)^{n} \\
& U(t)=1-(1+A t)^{-m} \\
& U(t)=1-B^{-\lambda t} \\
& U(t)=0,5\left[\left(1-e^{-\mu t}\right)^{a}+\left(1-e^{-\mu t}\right)^{b}\right] \\
& U(t)=c\left(1-e^{-v t}\right)+(1-c)\left(1-e^{-\xi t}\right)
\end{aligned}
$$

where $k, n, A, m, B, \lambda, \mu, a, b, c, v, \xi$-coefficients that are to be determined.

The representation of the damage function is carried out using expert methods [10] by 2 (fig. 1, a) or 3 (fig. 1, b) points. It's easy to notice that by 2 points: $U\left(t_{1}\right)=U_{1}$ и $U\left(t_{2}\right)=U_{2}$ the following coefficients may be obtained: $(k, n),(A, m)$ и $(B, \lambda)$ of the equations (1)-(3) respectively. Coefficients $(\mu, a, b)$ and $(c, v, \xi)$ of equations (4) and (5) - by 3 points: $U\left(t_{1}\right)=U_{1}, U\left(t_{2}\right)=U_{2}$ и $U\left(t_{3}\right)=U_{3}$ (fig. $\left.1, \mathrm{~b}\right)$.

Coefficients $(k, n),(A, m)$ и $(B, \lambda)$ can be determined from solutions of systems of two equations:

$$
\begin{aligned}
& \left\{\begin{array}{l}
U_{1}=\left[1-\exp \left(-k t_{1}\right)\right]^{n}, \\
U_{2}=\left[1-\exp \left(-k t_{2}\right)\right]^{n},
\end{array}\right. \\
& \left\{\begin{array}{l}
U_{1}=1-\left(1+A t_{1}\right)^{-m}, \\
U_{2}=1-\left(1+A t_{2}\right)^{-m},
\end{array}\right. \\
& \left\{\begin{array}{l}
U_{1}=1-B^{-\lambda t_{1}}, \\
U_{2}=1-B^{-\lambda t_{2}} .
\end{array}\right.
\end{aligned}
$$

Coefficients $(\mu, a, b)$ can be determined from solutions of systems of three equations:

$$
\left\{\begin{array}{l}
U_{1}=0,5\left[1-\exp \left(-\mu t_{1}\right)\right]^{a}+0,5\left[1-\exp \left(-\mu t_{1}\right)\right]^{b} \\
U_{2}=0,5\left[1-\exp \left(-\mu t_{2}\right)\right]^{a}+0,5\left[1-\exp \left(-\mu t_{2}\right)\right]^{b} \\
U_{3}=0,5\left[1-\exp \left(-\mu t_{3}\right)\right]^{a}+0,5\left[1-\exp \left(-\mu t_{3}\right)\right]^{b}
\end{array}\right.
$$

Coefficients $c, v, \xi$ can be determined similarly. 
Systems of equations (7)-(9) can be solved numerically [11], and in [9] a method for determining the coefficients $k$ и $n$ of equation (1) by two stages is presented. First, the relation $t_{2} / t_{1}=f_{1}(n)$ function is found as follows:

$$
\frac{t_{2}}{t_{1}}=\frac{\ln \left(1-U_{2}^{1 / n}\right)}{\ln \left(1-U_{1}^{1 / n}\right)}
$$

whence, by the known relation $t_{2 /} t_{1}$ the value $n$ is selected Then $k$ coefficient is being determined:

$$
k=-t_{1}^{-1} \ln \left(1-U_{1}^{1 / n}\right)=-t_{2}^{-1} \ln \left(1-U_{2}^{1 / n}\right)_{(11)}
$$

For the case when $U_{1}=0,1, U_{1}=0,5$ and $U_{2}=0,9$ the functions $f_{1}(n)$ are obtained, and they are presented in table 1 .

Table 1. Function $f(n)$ at $U_{2}=0,9$

\begin{tabular}{|c|c|c|c|c|c|c|c|c|c|}
\hline$n$ & 0,1 & 0,2 & 0,3 & 0,4 & 0,5 & 0,6 & 0,8 & 1 & $U_{1}$ \\
\hline \multirow{2}{*}{$\frac{t_{2}}{t_{1}}$} & $4,3 \cdot 10^{9}$ & 89279 & 2621 & 469,1 & 165,24 & 59,84 & 36,152 & 21,85 & 0,1 \\
\cline { 2 - 10 } & 438,8 & 28,12 & 11,646 & 7,520 & 5,773 & 4,827 & 3,836 & 3,322 & 0,5 \\
\hline \hline$n$ & 1,5 & 2 & 3 & 5 & 10 & 20 & 50 & 100 & $U_{1}$ \\
\hline \multirow{2}{*}{$\frac{t_{2}}{t_{1}}$} & 13,163 & 7,812 & 5,396 & 3,863 & 2,882 & 2,366 & 1,988 & 1,812 & 0,1 \\
\cline { 2 - 10 } & 2,707 & 2,419 & 2,133 & 1,893 & 1,686 & 1,553 & 1,438 & 1,378 & 0,5 \\
\hline
\end{tabular}

Note: at $U_{1}=0,5: f(29)=1,5 ; f(3,88)=2 ; f(1,204)=3 ; f(0,577)=5 ; f(0,3286)=10$.

For example, appearance of the damage fuction (1), presented at $U\left(t_{1}=20\right)=0,5$ and $U\left(t_{2}=30\right)=0,9$ using the equations (9), (10) and table 1 , is the following (curve 1 on fig. 2 ):

$$
U(t)=\left(1-e^{-0,1873 t}\right)^{29}
$$

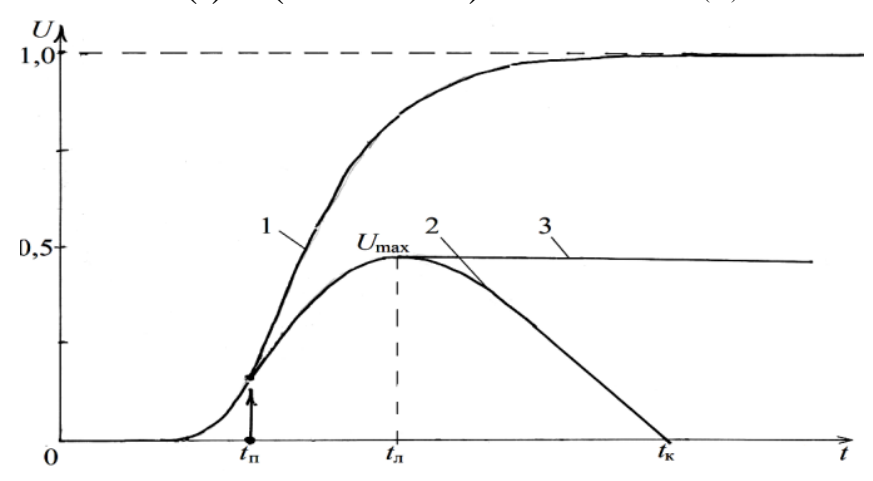

Fig. 2. The initial damage function (1) and its appearance in the elimination of the consequences, for example, of the release of hazardous chemicals (2) and when extinguishing a fire (3)

A similar approach is applicable to model (7), when the value of $m$ is determined by the relation $t_{2} / t_{1}=f_{2}(m)($ table 2$)$ :

$$
\frac{t_{2}}{t_{1}}=\frac{\left(1-U_{2}\right)^{-1 / m}-1}{\left(1-U_{1}\right)^{-1 / m}-1}
$$

Table 2. Function $f_{2}(m)$ at $U_{1}=0,5$ and $U_{2}=0,9$

\begin{tabular}{|c|c|c|c|c|c|c|c|c|}
\hline$m$ & 1 & 2 & 3 & 5 & 10 & 100 & 1000 & $\infty$ \\
\hline$t_{2} / t_{1}$ & 9 & 5,20 & 4,441 & 3,934 & 3,607 & 3,348 & 3,324 & 3,322 \\
\hline
\end{tabular}


However, there is a limitation corresponding to the condition $m \rightarrow \infty$ :

$$
\min \frac{t_{2}}{t_{1}}=\frac{\lg \left(1-U_{2}\right)}{\lg \left(1-U_{1}\right)}
$$

Applied to equation (4) using numerical methods [11] from solving the equations system (9) for the case $U(10)=0,1, U(20)=0,5$ and $U(30)=0,9$ the following values of coefficients are determined $\mu=0,2228, a=14,12$ and $b=161,1$, and as a result:

$$
U(t)=0,5\left(1-e^{-0,2228 t}\right)^{14,12}+0,5\left(1-e^{-0,2228 t}\right)^{161,1}
$$

\section{Results and discussion}

Using the damage function, it is possible to solve the problem of assessing the effectiveness of eliminating an emergency situation. In this case, two situations should be distinguished when at the moment $t_{l}$ the damage reaches its maximum $U_{\max }$ and remains at this level for some time (for example, a fire) and when after reaching $U_{\max }$ the damage is completely eliminated after the time $t_{\kappa}$, i.e. $U\left(t_{\kappa}\right)=0$, which may correspond to the neutralization of the release of an emergency chemical hazardous substance. Graphically similar situations are shown in Fig. 2.

If we assume that the elimination of an abnormal situation will begin in time $t_{p}$ after its occurrence and will occur at a rate of $k_{l}$ (can be determined by expert methods [10]), then the dynamics of damage is shown in Fig. 3, provided that equation (12) is valid.

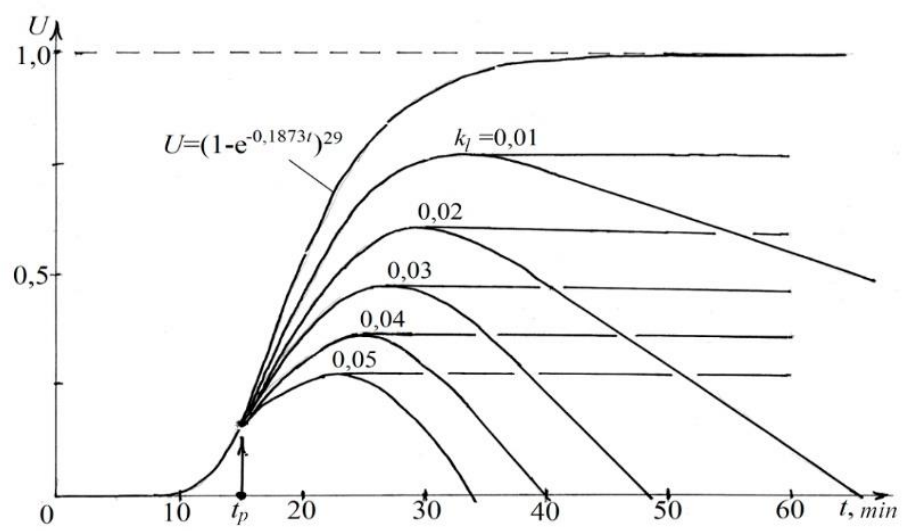

Fig. 3. An example of a damage function during actions to localize and eliminate an emergency situation

Taking into account the actions to localize and eliminate the contingency situation, the dynamics of damage $U(t)$ can be described in equation:

$$
U(t)=\left\{\begin{array}{l}
U_{0}(t) \text { when } t<t_{p}, \\
U_{0}(t)-k_{l}\left(t-t_{p}\right) \text { when } t \in\left[t_{p}, T\right), \\
U_{k} \text { when } t>T,
\end{array}\right.
$$

where $U_{0}$ - is an initial damage function determined by (1)-(5); $T=t_{1}$ and $U_{\mathrm{K}}=U_{\mathrm{max}}$, when, for example, fire extinguishing takes place; $T=t_{\mathrm{K}}$ and $U_{\mathrm{K}}=0$, when, for example, there is a neutralization of the release of hazardous chemicals.

Time $t_{1}$ can be found from the solution of the transcendental equation: 


$$
\frac{d U_{0}}{d t}=k_{l}
$$
[9]:

If the damage function is described by expression (1), then equation (17) takes the form

$$
k_{l}=k n E(1-E)^{n-1}
$$

where $E=\exp \left(-k\left(t_{1}-t_{\mathrm{p}}\right)\right)$.

Maximal damage $U_{\max }=U\left(t_{1}\right)$ at the initial damage function (1) can be determined from the equation (16):

$$
U_{\max }=\left(1-e^{-k t_{t}}\right)^{n}-k\left(t_{l}-t_{p}\right)
$$

Equations (18) and (19) can be solved numerically [11].

Time $t_{\mathrm{K}}$ can be found from the solution of the transcendental equation:

$$
U_{0}\left(t_{k}\right)=k_{l}\left(t_{k}-t_{p}\right)
$$

As an example, Fig. 3 shows function (12) and damage reduction functions at different speeds $k_{l}$ from 0.01 to $0.05 \mathrm{~min}^{-1}$.

With the help of the damage function, it becomes possible to solve the problem of comparing various methods of neutralizing an abnormal situation at an object, for example, a fire in a room when using automatic fire extinguishing installations (AFEI) [12]. In Fig. 4, using the damage function, it is clearly shown that with an increase in the operational efficiency of the AFEI by the value of $\Delta t$, the direct damage from the fire can be reduced by the value of $\Delta U$. Specific values of $\Delta t$ and $\Delta U$ can be obtained using the damage function for specific objects and specific types of AFEI.

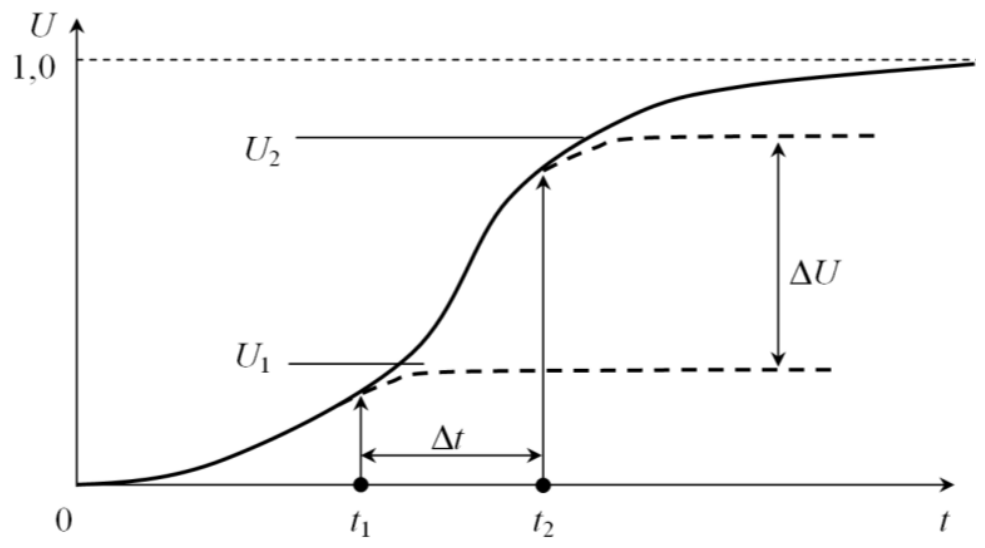

Fig. 4. An example of reducing damage at an object while increasing the response time of the localization system and eliminating an emergency situation

\section{Conclusion}

Thus, if the facility has a risk of various emergency situations (for example, fires), the above methods for constructing the damage function make it possible to objectively assess the 
characteristic time parameters of damage localization, taking into account the onset and speed of action of the corresponding systems, for example, AFEI.

Also, the problem of comparing two or more ways to minimize damage at a specific object can be solved, which will allow to objectively choose the best of them.

\section{References}

1. Federal Law «On Industrial Safety of Hazardous Production Facilities» dated 21.07.1997 No. 116-FZ (as amended).

2. The combat regulations of fire protection units, which determine the procedure for organizing fire extinguishing and carrying out emergency rescue operations. Approved. by order of the Ministry of Emergency Situations dated 16.10.2017, No. 444, registered with the Ministry of Justice of the Russian Federation on 20.02.2018, reg. No. 50100.

3. Methodical recommendations for drawing up plans for extinguishing fires and fire extinguishing cards. Letter of the Ministry of Emergency Situations of Russia dated 01.03.2013 No. 43-956-18.

4. Decree of the Government of the Russian Federation of 15.09.2020 No. 1437 «On approval of the Regulation on the development of action plans for the containment and elimination of the consequences of accidents at hazardous production facilities».

5. Decree of the Government of the Russian Federation of December 31, 2020 No. 2451 «On approval of the Rules for organizing measures for the prevention and elimination of oil and oil products spills on the territory of the Russian Federation, with the exception of the internal sea waters of the Russian Federation and the territorial sea of the Russian Federation, as well as on the recognition lost the force of some acts of the Government of the Russian Federation».

6. Methodology for determining the calculated values of fire risk at production facilities. Approved. by order of the Ministry of Emergency Situations dated 10.07.2009, No. 404, registered with the Ministry of Justice of the Russian Federation on 17.08.2009, reg. No. 14541.

7. Methods for determining the calculated values of fire risk in buildings, structures and structures of various classes of functional fire hazard. Approved. by order of the Ministry of Emergency Situations dated 30.05.2009, No. 382, registered with the Ministry of Justice of the Russian Federation on 05.08.2009, reg. No. 14486.

8. G.I. Abduragimov, Tarantsev A.A., The theory of queuing in the management of fire protection. Monograph. Moscow: Academy of State Fire Service of the Ministry of Internal Affairs of Russia, 2000, 102 p.

9. A.D. Ishchenko, Krymsky V.V., Tarantsev Andr.A., On the construction of the damage function, Problems of risk management in the technosphere, No. 4 (56), 2020, pp.5459.

10. A.N. Anokhin, Expert assessment methods, Tutorial. - Obninsk: IATE, 1996, 148 p.

11. R.V. Hemming, Numerical Methods for Scientists and Engineers, Moscow: Nauka, $1972,400 \mathrm{p}$.

12. SP 485.1311500.2020 SPPZ. Automatic fire extinguishing installations. Norms and rules of design. 